\title{
Impact of COVID-19 on dermatologic surgery: experience of a Midwestern academic practice
}

\author{
Remi Hamel $^{1} \cdot$ Bruin Pollard $^{2} \cdot$ M. Laurin Council ${ }^{1}$ (i)
}

Received: 20 March 2021 / Revised: 16 September 2021 / Accepted: 24 September 2021 / Published online: 10 October 2021

(c) The Author(s), under exclusive licence to Springer-Verlag GmbH Germany, part of Springer Nature 2021

\begin{abstract}
The novel coronavirus disease 2019 (COVID-19) pandemic has disrupted clinical practice everywhere. The aim of this study was to quantify the specific impact of COVID-19 on skin cancer treatment at an academic dermatologic surgery practice. We conducted a retrospective chart review to compare metrics such as patient visits, histological upgrading, and Mohs stages per tumor between 60-day periods immediately before and after COVID-19 was declared a pandemic. Out of 1138 total encounters, decreases of 58\% in total in-person visits and 38\% in Mohs surgeries performed were observed following declaration of the pandemic. More squamous cell carcinoma and squamous cell carcinoma in situ (SCC/SCCIS) and less basal cell carcinoma (BCC) tumors were treated post-declaration compared to pre-declaration. There was a significantly higher histological upgrade rate for total tumors, as well as for the BCC subgroup, but not the SCC/SCCIS subgroup. While the overall number of dermatologic surgeries decreased after declaration of the pandemic, the higher histological upgrade rate reflects an appropriate triage of higher risk skin cancers. These findings may be useful both to assess the effectiveness of protocols for COVID-19 and to prepare for future resource-limited scenarios.
\end{abstract}

Keywords COVID-19 $\cdot$ Pandemic $\cdot$ Dermatologic surgery $\cdot$ Mohs surgery

\section{Introduction}

The World Health Organization (WHO) declared COVID19 a pandemic on March 11, 2020. The following week, the Centers for Medicare and Medicaid Services (CMS) requested that "elective surgeries, non-essential medical, surgical, and dental procedures be delayed" [2]. Likewise, the National Comprehensive Cancer Network (NCCN) issued an advisory statement for non-melanoma skin cancer care [5]. Adapting to these recommendations required radical protocol adjustments in dermatologic surgery offices, including only scheduling patients with high-risk/ symptomatic tumors or with specific comorbidities, as well as switching to telemedicine consultations. While the general impact of the COVID-19 pandemic has been widely

M. Laurin Council

mcouncil@wustl.edu

1 Division of Dermatology, Department of Medicine, Washington University School of Medicine, 660 South Euclid Avenue, Saint Louis, MO 63110, USA

2 Washington University School of Medicine, Saint Louis, MO, USA recognized, quantifying specific impact on dermatologic surgery practices in the United States may help inform triage decisions in future.

\section{Materials and methods}

We performed a retrospective chart review on all patients who underwent Mohs surgery at the Washington University Center for Dermatologic and Cosmetic Surgery West County office during the 60-day period before and after March 11, 2020. Period 1 (pre-pandemic declaration) was from $1 / 11 / 20$ to $3 / 10 / 20$ (60 days), and period 2 (post-pandemic declaration) was from $3 / 11 / 20$ to $5 / 9 / 20$ (60 days). Metrics included number of surgeries performed, time from initial biopsy to surgery, stages per tumor, and incidence of histological upgrading. NCCN guidelines on risk factors for local recurrence or metastases informed histologic upgrading. For squamous cell carcinoma (SCC), high-risk pathological features included poorly differentiated tumors, acantholytic, adenosquamous, desmoplastic, or metaplastic subtypes, $>6 \mathrm{~mm}$ thickness or invasion beyond subcutaneous fat, and perineural, lymphatic, or vascular involvement. For 
Table 1 Characteristics of skin cancers treated during periods 1 vs. 2

\begin{tabular}{|c|c|c|c|c|}
\hline & & Period $1(n=372)$ & Period $2(n=231)$ & $p$ value \\
\hline \multirow{7}{*}{$\begin{array}{l}\text { Total } \\
(n=603)\end{array}$} & Upgraded tumors & $23(6.2 \%)$ & $25(10.8 \%)$ & 0.0407 \\
\hline & Days (IQR) from initial biopsy to Mohs surgery & $79(59-107)$ & $66(41-107)$ & 0.0085 \\
\hline & Number of Mohs stages per tumor: & & & 0.0016 \\
\hline & 1 & $195(52.4 \%)$ & $150(64.9 \%)$ & \\
\hline & 2 & $131(35.2 \%)$ & $65(28.1 \%)$ & \\
\hline & 3 & $31(8.3 \%)$ & $6(2.6 \%)$ & \\
\hline & $4-6$ & $15(4.0 \%)$ & $10(4.3 \%)$ & \\
\hline \multirow{7}{*}{$\begin{array}{l}\text { BCC } \\
(n=260)\end{array}$} & Upgraded tumors & $17(9.4 \%)$ & $22(27.9 \%)$ & 0.0001 \\
\hline & Days (IQR) from initial biopsy to Mohs surgery & $99(74-114)$ & $101(63-127)$ & 0.8396 \\
\hline & Number of Mohs stages per tumor: & & & 0.1817 \\
\hline & 1 & $72(39.8 \%)$ & $37(46.8 \%)$ & \\
\hline & 2 & $79(43.7 \%)$ & $33(41.8 \%)$ & \\
\hline & 3 & $19(10.5 \%)$ & $3(3.8 \%)$ & \\
\hline & $4-6$ & $11(6.1 \%)$ & $6(7.6 \%)$ & \\
\hline \multirow{7}{*}{$\begin{array}{c}\text { SCC/SCCIS } \\
(n=334)\end{array}$} & Upgraded tumors & $6(3.2 \%)$ & $3(2.0 \%)$ & 0.7363 \\
\hline & Days (IQR) from initial biopsy to Mohs surgery & $65(48-90)$ & $50(26-93)$ & 0.0154 \\
\hline & Number of Mohs stages per tumor: & & & 0.0827 \\
\hline & 1 & $120(64.9 \%)$ & $112(75.2 \%)$ & \\
\hline & 2 & $51(27.6 \%)$ & $30(20.1 \%)$ & \\
\hline & 3 & $11(6.0 \%)$ & $3(2.0 \%)$ & \\
\hline & $4-6$ & $3(1.6 \%)$ & $4(2.7 \%)$ & \\
\hline
\end{tabular}

$B C C$ basal cell carcinoma, SCC squamous cell carcinoma, SCCIS squamous cell carcinoma in situ, IQR interquartile range

basal cell carcinoma (BCC), high-risk pathological features included aggressive growth patterns (infiltrative, micronodular, morpheaform, basosquamous, sclerosing, or carcinosarcomatous differentiation) or perineural involvement [6]. The study was granted exemption by the Washington University Institutional Review Board (\#202,005,146) and approved by the Siteman Cancer Center Protocol Review and Monitoring Committee.

Data are presented as median with interquartile range for quantitative variables, and frequencies with percentages for qualitative variables. Wilcoxon rank sum test was performed to compare numeric variables between two periods. Chi-square test or Fisher's exact test was used to detect the association between two categorical variables. A two-sided $p$ value less than or equal to 0.05 was considered as statistically significant. Data were analyzed using SAS $^{\circledR}$ (SAS Institute Inc., Cary, NC, USA) 9.4 version.

\section{Results}

There were 1138 total in-person patient visits: 800 during period 1 (before declaration of pandemic) versus 338 during period 2 (after declaration of pandemic). Mohs surgery was performed on 32 out of 60 days in period 1 and 29 out of 60 days in period 2.
A total of 603 tumors (260 BCC, 334 SCC/SCCIS, and 9 other) were treated with Mohs surgery: 372 in period 1 versus 231 in period 2. This difference represents a $38 \%$ relative decrease in the number of tumors treated (Table 1). By Fisher's exact test, there was a significant change in the overall distribution of tumors treated between period 1 (181 (48.7\%) BCC, 185 (49.7\%) SCC/SCCIS, and 6 (1.6\%) other) and period 2 (79 (34.2\%) BCC, 149 (64.5\%) SCC/ SCCIS, and $3(1.3 \%)$ other) $(p=0.0018)$. Period 2 had a significantly higher histological upgrade rate for total tumors (10.8\% vs. $6.2 \%, p=0.0407)$. This was also true in the BCC subgroup $(27.9 \%$ vs. $9.4 \%, p=0.0001)$, but not the $\mathrm{SCC} /$ SCCIS subgroup.

Comparing pre-op to post-op tumor size (all tumor types), there was an increase in mean area from 0.9 to 2.8 $\mathrm{cm}^{2}$ (210\% increase) in period 1 and 1.3 to $3.0 \mathrm{~cm}^{2}(140 \%$ increase) in period 2 . The number of cases with subclinical spread (defined as greater than 3 stages) was 15 in period 1 and 10 in period 2 . One tumor in period 1 and two tumors in period 2 had perineural invasion noted at time of Mohs surgery. Using the Chi-square test, the overall distribution of Mohs stages per tumor was significantly different between periods for total tumors $(p=0.0016)$. Period 2 had a higher percentage of one-stage Mohs procedures, while period 1 had higher percentages of two- and three-stage Mohs procedures. Both periods had similar percentages of four- to 
six-stage procedures. The BCC and SCC/SCCIS subgroups each showed similar distributions of stages between periods, but the differences were not statistically significant.

The median number of days from initial biopsy to Mohs surgery was significantly reduced in period 2 compared to period 1 for total tumors (79 vs. $66, p=0.0085$ ) as well as the SCC/SCCIS subgroup (65 vs. 50, $p=0.0154$ ).

\section{Discussion}

Our practice saw the total number of in-person visits decreased by greater than $50 \%$, and Mohs surgeries by $38 \%$, following declaration of the COVID-19 pandemic. Non-essential visits such as cosmetics were canceled, while others such as consults and follow-ups were converted to telemedicine. Our clinic deferred treatment of lower risk $\mathrm{BCC}$, and those treated had a higher histological upgrade rate, reflecting appropriate triage of high-risk BCC tumors. Relatively more SCC, which have higher risk of metastasis than BCC, were treated during the pandemic (either invasive SCC, or in situ for which initial biopsy could not equivocally rule out invasive disease). These changes are consistent with similar studies conducted in the United Kingdom and Massachusetts, highlighting a global effort to prioritize necessary care for high-risk skin cancer while also managing the risk of virus transmission [1,3].

Unlike BCC, the SCC/SCCIS subgroup did not show a higher histological upgrade rate. This is likely due to the fact that most of these tumors had an initial pathology diagnosis of "SCCIS, cannot rule out invasive SCC," but ultimately Mohs surgery did not identify invasive SCC. This explanation may also account for the fact that period 2 had a higher percentage of single-stage Mohs procedures than period 1. In fact, another study recently reported that only $10 \%$ of indeterminate SCCIS tumors harbor invasive SCC, while the rest remain SCCIS or are found to be without evidence of neoplasm upon further evaluation [4]. Overall, these findings support the idea that $\mathrm{BCC}$ tumors are more accurately triaged into risk groups than SCC/SCCIS tumors, given the difference in upgrade rate. However, more data are needed to confirm whether BCC tumors deferred during the COVID19 pandemic did indeed have a lower upgrade rate.

Our results suggest that it is reasonable to consider deferring treatment of indeterminate SCCIS tumors when resources are limited, such as during a pandemic. Our practice, which was fortunate enough to continue offering Mohs surgery for tumors in this subgroup, demonstrates the effectiveness of NCCN recommendations for triage in the COVID-19 cohort of skin cancers. Our data may serve as a useful benchmark for comparison in future resource-limited scenarios, or in case, the COVID-19 pandemic lingers due to additional waves of variant strains, vaccine hesitancy, or other dynamic challenges.

Author contributions RH and MLC designed the study. RH performed data collection. BP performed a literature review. $\mathrm{RH}$ and BP drafted and revised the manuscript. MLC critically reviewed and revised the manuscript.

Funding None.

Availability of data and material Not applicable.

Code availability Not applicable.

\section{Declarations}

Conflict of interest Dr. Council is a board member and consultant for Sanifo Genzyme and Regeneron, which is not relevant to the study.

Ethics approval The study was granted exemption by the Washington University Institutional Review Board (\#202005146) and approved by the Siteman Cancer Center Protocol Review and Monitoring Committee.

Research involving humans and/or animal participants Not applicable.

Consent to participate Not applicable.

Consent for publication Not applicable.

\section{References}

1. Capitelli-McMahon H, Hurley A, Pinder R, Matteucci P, Totty J (2021) Characterising non-melanoma skin cancer undergoing surgical management during the COVID-19 pandemic. J Plastic Reconstr Aesth Surg JPRAS 74(3):644-710. https://doi.org/10. 1016/j.bjps.2020.10.042

2. Centers for Medicare and Medicaid Services. "Non-Emergent, Elective Medical Services, and Treatment Recommendations." www.cms.gov/files/document/cms-non-emergent-elective-medic al-recommendations.pdf. Accessed 10 May 2020

3. Danesh MJ, Porter M, Brag K, Salian P, Olbricht S (2021) COVID-19 impacts on dermatologic surgery patients: a single institution experience. J Am Acad Dermatol. https://doi.org/10. 1016/j.jaad.2021.02.060

4. Knackstedt TJ, Brennick JB, Perry AE, Li Z, Quatrano NA, Samie FH (2015) Frequency of squamous cell carcinoma (SCC) invasion in transected SCC in situ referred for Mohs surgery: the Dartmouth-Hitchcock experience. Int J Dermatol 54(7):830-833. https://doi.org/10.1111/ijd.12867

5. National Comprehensive Cancer Network. "Advisory Statement for Non-Melanoma Skin Cancer Care During the COVID-19 Pandemic." www.ncen.org/covid-19/pdf/NCCN-NMSC.pdf. Accessed 17 May 2020

6. National Comprehensive Cancer Network. "Skin Cancer Guidelines." www.nccn.org/professionals/physician_gls/pdf/. Accessed 17 May 2020

Publisher's Note Springer Nature remains neutral with regard to jurisdictional claims in published maps and institutional affiliations. 